Open Access

\title{
A multivariate analysis on the comparison of raw notoginseng (Sanqi) and its granule products by thin-layer chromatography and ultra-performance liquid chromatography
}

Xian Zhou ${ }^{1 \dagger}$, Valentina Razmovski-Naumovski ${ }^{1,2+}$ and Kelvin Chan ${ }^{1,2^{*}}$

\begin{abstract}
Background: Granule products produced from medicinal herbs are gaining popularity. However, there have been few studies comparing the quality or efficacy of granules with those of herbal formulations. This study aims to compare commercially available notoginseng (Sanqi in Chinese) in both raw and granule forms by thin layer chromatography (TLC) and ultra-performance liquid chromatography with photodiode array detection (UPLC-PDA) using multivariate analysis.

Methods: Aqueous extracts of the raw herb (collected from six different sources in China) and granule products (purchased in China, Taiwan and Australia) were re-extracted with methanol to remove water-soluble excipients. Five compounds (ginsenosides Rg1, Rg2, Rd and Rb1 and notoginsenoside NR1) in the methanolic extracts were quantified by TLC and UPLC-PDA. Multivariate statistical analysis using hierarchical component analysis (HCA) and principal component analysis (PCA) was used to determine the similarities between the granule products and raw herbs. A 2,2'-azino-bis(3-ethylbenzothiazoline-6-sulfonic acid) (ABTS) assay was used to measure the antioxidant capacities of the extracts.

Results: HCA and PCA of the TLC analysis clustered the granule products into one group. By UPLC analysis, the raw herbs and two of the granule products (G7 and G12) were allocated into Group 1 and the rest of the granule products into Group 2. The contents of the five marker compounds in Group 1 were higher than Group 2 and also exhibited stronger ABTS activity $(P=0.005)$. By Pearson correlation, the contents of the five compounds in the samples were positively and significantly correlated to their antioxidant activities.

Conclusions: UPLC was more efficient than TLC for the simultaneous determination of the five major compounds in Sanqi products in terms of linearity, higher sensitivity and repeatability. The statistical analysis of the samples by HCA and PCA revealed that the contents of the marker compounds were significantly higher in the raw herb group than the granule group.
\end{abstract}

\section{Background}

The use of herbal preparations as pharmaceutical products is considered convenient, portable and consistent [1]. Granule products of herbal preparations are increasingly popular among consumers. Granule products for use in traditional Chinese medicine are prepared by concentrating a herbal extract to a dry powder, and then

\footnotetext{
* Correspondence: k.chan@uws.edu.au

${ }^{\dagger}$ Equal contributors

${ }^{1}$ The National Institute of Complementary Medicine, University of Western Sydney, Locked Bag 1797, Penrith, NSW 2751, Australia

${ }^{2}$ Faculty of Pharmacy, The University of Sydney, Sydney, Australia
}

adding excipients such as starch, dextrins, lactose and soluble fibres [1]. As with pharmaceutical preparations, these products must meet minimum quality, safety and efficacy requirements, especially testing the pharmacological efficacy of granule products against decoctions prepared by boiling medicinal herbs in water. Non-standardised manufacturing and quality control procedures would inadvertently introduce clinical inconsistency in dosages for consumers. Currently, there are no established regulatory guidelines for the standardisation of granule products, and few studies have compared the granule's chemical profile to that of the raw material. Thus, the quality assurance/

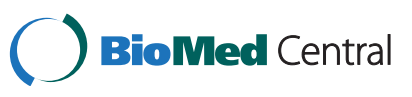


control of granule products is a critical issue for their future development.

Thin layer chromatography (TLC) and high performance liquid chromatography are standard industry methods for the quality control of herbal products [2-4]. TLC has been used extensively in industry because of its simplicity, low cost and versatility for simultaneous analysis of multiple samples [5]. In the last decade, ultraperformance liquid chromatography (UPLC) has become the preferred method for the analysis of herbal products because of its sensitivity and high resolution for the quantification of active components [5-7].

Multivariate statistical analyses such as hierarchical component analysis (HCA) and principal component analysis (PCA) are employed to compare multiple samples [6]. HCA classifies samples into clusters according to their similarities or differences. PCA uses a linear mathematical algorithm to derive principal components (PCs), and assesses how certain combinations of key factors account for differences between samples [8].

Notoginseng (Sanqi) is the root and rhizome of Panax notoginseng (Burk.) F. H. Chen. There is a large market for notoginseng granules in China, with data from the China Food and Drug Administration showing more than 50 granule products are currently manufactured [9]. These products are also widely available in Australia and other Western countries. Therefore, Sanqi was chosen as an example for the quality control of granule products. Sanqi is commonly used for the management of cardiovascular complications [10] because of its antioxidant, antiplatelet [11], haemostatic [12] and fibrinolytic activity [13]. The plant-based antioxidant effects were involved in the prevention and treatment of cardiovascular diseases $[14,15]$. The antioxidant activities of the extracts of raw notoginseng and its saponin compounds have been well studied [10]. The antioxidant capacities of notoginseng products can be measured using the simple 2,2'-azino-bis(3-ethylbenzothiazoline6-sulfonic acid (ABTS) assay, and the results used to assess the claimed bioactivity. However, no studies have assessed the antioxidant effects of granule formulations in comparison with the raw herb.

This study aims to compare commercially available notoginseng (Sanqi) in both raw and granule forms by TLC and UPLC-photodiode array (PDA) using multivariate analysis. In this study, the ginsenosides Rg1, Rg2, Rd and Rb1 and notoginseng R1 (NR1) (Additional file 1) were qualitatively and quantitatively evaluated in raw notoginseng and granule products using TLC. The results were compared with those from UPLC with PDA detection, and the quantitative data were analysed by HCA and PCA. The ABTS assay was used to determine the antioxidant capacities of the samples and examine any correlation of this to the amount of the marker compounds.
The results from this in vitro chemical assay, when correlated to the chemical profiles of the samples, will provide further information about the quality of the notoginseng products.

\section{Methods}

\section{Chemicals and plant materials}

Raw notoginseng (R1-R6) was collected from six different sources in China and twelve herbal granule products (G1-G12) were purchased in China, Taiwan and Australia (Additional file 2). The raw herb samples were authenticated by Professor Si-bao Chen (Department of Applied Biology and Chemical Technology, Hong Kong Polytechnic University, Hong Kong, China) according to the Hong Kong Materia Medica Standards and Pharmacopoeia of the People's Republic of China 2010 (PPRC). A voucher specimen of each sample was deposited in the National Institute of Complementary Medicine (NICM), University of Western Sydney. Product names have been omitted due to the absence of consent for disclosure. The five marker compounds, notoginseng NR1 and ginsenosides Rg1, Rb1, $\mathrm{Rd}$ and $\mathrm{Rg} 2$, were purchased from Chengdu Biopurify Phytochemicals Ltd. (Chengdu, China; purity $>98 \%$ ). HPLC grade acetonitrile and methanol were purchased from Thermo Fisher Scientific (Waltham, MA, USA). Analytical grade ethyl acetate, sulfuric acid and chloroform were purchased from Ajax Finechem (Sydney, Australia).

\section{Sample preparation}

The manufacturing process for granule products was considered an industrial-scale reproduction of a water decoction. In this study, $1 \mathrm{~g}$ of a ground raw herb sample (30-mesh size) was refluxed in $30 \mathrm{~mL}$ of boiling water three times for $30 \mathrm{~min}$. The aqueous extracts were combined then centrifuged at $672 \times g$ for $5 \mathrm{~min}$ and evaporated to dryness. Each aqueous raw herb extract and $1 \mathrm{~g}$ of each granule sample was sonicated in $10 \mathrm{~mL}$ of methanol for $30 \mathrm{~min}$ (done three times), followed by centrifugation at $672 \times g$ for $5 \mathrm{~min}$. The supernatants were collected and evaporated to dryness at $60{ }^{\circ} \mathrm{C}$ under vacuum. This procedure separated the water-soluble excipients from the granule samples, and allowed for comparison of the two products as methanol extracts (Additional file 3). The dry residue was weighed and re-dissolved in a minimum volume of methanol for further analysis.

\section{Preparation of standard solutions}

Stock standard solutions of the reference marker compounds Rg1, Rg2, Rd, Rb1 and NR1 ( $2 \mathrm{mg} / \mathrm{mL})$ were prepared in methanol and stored at $4{ }^{\circ} \mathrm{C}$. Working standard solutions for calibration at five different concentrations were freshly prepared by dilution of the stock solutions. The concentration ranges of the calibration curves for 
TLC and UPLC were $0.05-1 \mathrm{mg} / \mathrm{mL}$ and $0.0125-1 \mathrm{mg} / \mathrm{mL}$, respectively.

\section{Instrumentation and chromatographic conditions TLC}

A TLC kit (CAMAG Chemie-Erzeugnisse \& Adsorptionstechnik AG, Muttenz, Switzerland) containing a Linomat 5 automatic applicator with $100-\mu \mathrm{L}$ syringes and a softwarelinked (winCATs ver.1.3.0 system) imaging device was used for TLC. TLC plates were evaluated using a CAMAG Scanner 3 with visible light, $366 \mathrm{~nm}$ light and $254 \mathrm{~nm}$ light, and a camera (Canon PSG $\times$ digital camera). The TLC plates were silica gel $60 \mathrm{~F}_{254}$ plate $(20 \mathrm{~cm} \times 20 \mathrm{~cm})$ (Merck KGaA, Darmstadt, Germany), and each plate was cut into $10 \mathrm{~cm} \times 10 \mathrm{~cm}$ squares before use. The application position was $10 \mathrm{~mm}$ from the lower edge of the TLC plate. All samples were applied according to the following settings: $8-\mathrm{mm}$ band width, 2-mm space between tracks, and eight tracks on each plate. Standards and samples $(6 \mu \mathrm{L})$ were loaded onto the TLC plates. All remaining measurement parameters were default settings. CAMAG Twin Trough chambers $(10 \times 10 \mathrm{~cm})$ with a stainless steel lid were used for the development of the plates with a mobile phase of chloroform-ethyl acetate-methanol-water (15:40:22:9, v/v/v/v) as described previously [16]. The chamber was kept in a fume hood at an ambient temperature of $20{ }^{\circ} \mathrm{C}$. The plate was developed vertically from the lower edge to $80 \mathrm{~mm}$. After development, the plate was air-dried for $10 \mathrm{~min}$ before derivatisation. Ginsenosides have weak absorption at low UV wavelengths as they do not possess a strong chromophore [17]. Therefore, the plates were dipped into ice cold sulfuric acid (10\% sulfuric acid in iced methanol) in a CAMAG chamber tank. The plates were then air-dried for $10 \mathrm{~min}$, and heated at $100{ }^{\circ} \mathrm{C}$ in an oven for $5 \mathrm{~min}$. The analytes were quantified with the CAMAG Scanner 3 using a D2\&W lamp set at $366 \mathrm{~nm}$, with $20-\mathrm{mm} / \mathrm{s}$ scanning speed. A CAMAG Reprostar 3 with winCATs software was used to analyse the derivatised plates.

\section{UPLC-PDA}

UPLC-PDA analyses were performed using a Waters ACQUITY UPLC ${ }^{\mathrm{rм}}$ system (Waters, Milford, USA), equipped with quaternary solvent manager (ACQ-QSM), quaternary pump, sampler manager FTN (ACQ-FTN), column compartment, PDA detector (ACQ-PDA), and connected to Waters Empower 3 software. UPLC separations were carried out using an Acquity UPLC BEH $\mathrm{C}_{18}$ column $(150 \mathrm{~mm} \times 2.1 \mathrm{~mm}, 1.7 \mu \mathrm{m})$ with an attached pre-column $(2.1 \mathrm{~mm} \times 5 \mathrm{~mm}, 1.7 \mu \mathrm{m})$ (Waters). The column and sample temperature were kept at $20{ }^{\circ} \mathrm{C}$ and $4{ }^{\circ} \mathrm{C}$, respectively. The initial mobile phase consisted of water (A)-acetonitrile (B) (82:18, v/v). Gradient conditions were based on a modification of a method described previously [18], with a gradient elution as follows: 0$5.5 \mathrm{~min}, 18-19 \% \mathrm{~B}$; 5.5-6.0 min, 19-31 \% B; 6.0-9.5 min, 31-35\% B; 9.5-12.0 min, 35-56 \% B, $100 \%$ B for $6 \mathrm{~min}$. The column was reconditioned isocratically with $18 \% \mathrm{~B}$ for $7 \mathrm{~min}$. The flow rate was $0.30 \mathrm{~mL} \mathrm{~min}{ }^{-1}$ and the injection volume was $1 \mu \mathrm{L}$. The detection wavelength was $203 \mathrm{~nm}$. All solutions were filtered using $0.2-\mu \mathrm{m}$ polytetrafluoroethylene membrane filters before injection. The total run time for the analysis was $25 \mathrm{~min}$. The identification of compounds in the samples was carried out by comparison of UV spectra and retention times.

\section{LC-MS}

The purity of the references was verified by liquid chromatography-mass spectrometry (LC-MS). LC-MS experiments were performed on a Waters Acquity Xevo TQ triple quadruple mass spectrometer coupled to a binary pump, PDA detector and an autosampler (Waters, Milford, USA). Mass spectra were acquired in negative electrospray ionisation (ESI) mode with a mass range of $m / z$ 100-1200. The data were analysed by MassLynx Mass Spectrometry software (Waters, Milford, USA).

\section{Validation procedure}

The TLC and UPLC methods were partially validated in terms of linearity and repeatability. Six-point calibration curves were constructed with linear ranges of 0.05$1 \mathrm{mg} / \mathrm{mL}$ and $0.0125-1 \mathrm{mg} / \mathrm{mL}$ for TLC and UPLC, respectively. Six replicates of the calibration standards were prepared, and each was analysed in triplicate. Regression equations, $y=\mathrm{a} x+\mathrm{b}$, were calculated, where $x$ and $y$ are the concentration of the reference samples and the peak area, respectively. The LOD and LOQ were calculated according to the equations, $\mathrm{LOD}=3.33 \times$ (standard deviation [SD] of $y$-intercept/mean of slope) and LOQ $=10 \times(\mathrm{SD}$ of $y$-intercept/mean of slope) [19]. The quantity of each analyte was obtained from the corresponding calibration curve. The relative standard deviation (RSD) was used as a measure of repeatability. The intra-day precision was evaluated by analysing four concentrations of each marker compound three times within a day, and the inter-day reproducibility was examined on three consecutive days.

\section{ABTS antioxidant assay}

A modification of an established procedure [20] was used to estimate the ABTS radical scavenging capacities of the notoginseng extracts. The ABTS radical working solution was prepared by mixing equal volumes of $7 \mathrm{mmol} / \mathrm{L}$ ABTS solution with $2.45 \mathrm{mmol} / \mathrm{L}$ potassium persulfate solution, and the mixture was left in the dark for 12 to $16 \mathrm{~h}$ at room temperature. On the day of analysis, the stock solution was diluted with PBS ( $\mathrm{pH} 7.4$ ) until an initial absorbance value of 0.4 at $730 \mathrm{~nm}$ was reached. Diluted ABTS $(200 \mu \mathrm{L})$ was mixed with $20 \mu \mathrm{L}$ 
of the sample or standard and the absorbance reading was taken $5 \mathrm{~min}$ after mixing. Trolox $(0.045-0.330 \mathrm{mmol} / \mathrm{L})$ was used as the standard. The antioxidant activity was calculated as the concentration of $\mathrm{ABTS}^{+}$quenched by $1 \mathrm{mmol} / \mathrm{L}$ of Trolox. The antioxidant activities of the notoginseng samples were expressed as Trolox equivalents per dry weight (DW) of the sample $\left(\mathrm{mmol} \mathrm{L}^{-1} / \mathrm{g}\right.$ of DW) [21]. The ABTS assay was performed in triplicate.

\section{Statistical analysis}

The results from the granules were converted back to raw herb values, by the ratio specified on the product, to compare the raw herb and granule products. Here, the residue obtained from the granules after the methanol extraction was assumed to be equivalent to the raw herb water extract without excipients. The yields were expressed as the mean \pm SD of three extractions. Each of the three extracts from the same sample were analysed three times by TLC and UPLC, with the final quantitative results from TLC and UPLC instrumental analyses expressed as the mean \pm SD. Quantitative results were reported as milligrams per grams of the DW of the raw herb $(\mathrm{mg} / \mathrm{g})$.

The TLC and UPLC quantitative data were analysed non-parametrically by IBM SPSS Statistics 20 for Windows (SPSS Inc., Chicago, IL, USA) to determine any significant differences in the marker compound contents between the raw herbs and granules. The compound(s) that showed significant differences were assigned as variables for HCA. The data were pretreated by autoscaling. HCA was conducted by the Ward's method and Euclidean distances. The results were expressed as dendrograms where the length of the branches between samples reflected the degree of similarity between them.

PCA was conducted by XLSTAT (Addinsoft, New York, USA). PCA converted the original variables (five marker compounds) into a new set of linearly uncorrelated factors (PCs). The first and second PCs corresponded to the largest possible variance of the original variables. The results were represented in a biplot (score plot and loading plot), which showed the distribution of the samples and the correlation of the five original variables to the two PCs $[8,22]$.

The yield and ABTS results were analysed by independent samples $t$-test and non-parametric analysis. Pearson correlation coefficients $(r)$ indicated the strength of the correlation between the contents of the five marker compounds in the samples and their ABTS scavenging activities. These analyses were conducted by SPSS, and $P<0.05$ was considered statistically significant.

\section{Results and discussion Extraction yield}

The average amount of dry residue obtained from each sample is shown in Table 1 . The average yields of the raw herbs (R1-R6) were consistent at $21.07 \pm 3.39 \%$ of
Table 1 The average yields of the raw herbs (R1-R6) and granule (G1-G12) methanol extracts ( $n \geq 3$ )

\begin{tabular}{lcc}
\hline Samples & Average yield (\%) & RSD $(\%)^{a}$ \\
\hline R1 & 24.17 & 8.65 \\
R2 & 23.52 & 4.34 \\
R3 & 23.26 & 0.13 \\
R4 & 19.82 & 6.71 \\
R5 & 15.150 & 1.06 \\
R6 & 20.50 & 5.71 \\
G1 & 3.59 & 11.75 \\
G2 & 8.58 & 7.44 \\
G3 & 9.17 & 13.02 \\
G4 & 3.16 & 7.39 \\
G5 & 3.12 & 22.98 \\
G6 & 2.02 & 0.94 \\
G7 & 12.47 & 3.37 \\
G8 & 1.07 & 9.01 \\
G9 & 7.62 & 6.83 \\
G10 & 7.16 & 9.66 \\
G11 & 11.54 & 9.79 \\
G12 & 18.85 & 7.07 \\
\hline R & &
\end{tabular}

$R$ raw herb; $G$ granule

${ }^{\mathrm{a}} \mathrm{RSD}(\%)=100 \times$ S.D. $/$ mean

the dry weight of the herb. Independent samples $t$-tests illustrated that, within the raw herb group, the yield from R5 was significantly lower than those from the rest of the raw herbs $(P=0.029)$. By contrast, the average yields of the granule products were variable and ranged from $1.07-18.85 \%$. The differences in the yield for the granule products could be caused by the varied manufacturing processes used by the companies that produced the granules. For example, extraction procedures, temperature conditions, the type and amount of excipients used, and other factors in the manufacturing processes can affect the quality of the finished product. A comparison of the two groups using non-parametric analysis showed that the yields from the raw herbs were significantly higher than those from the granules $(P=0.000)$. The excipients added to the granules during manufacturing might be slightly soluble in methanol, and may have affected the final calculation of the notoginseng content of the granule product thus, giving a lower concentration for the granule extract. The RSD yields for the raw herbs ranged from $0.19-8.66 \%$, whereas the RSD yields for the granules ranged from $0.95-23.014 \%$, indicating that the granule particles were not uniform, even though every granule bottle was shaken before sampling to redistribute the particles. 
Table 2 Regression data, detection/quantification limits and precision data for the five compounds determined by UPLC-PDA

\begin{tabular}{|c|c|c|c|c|c|c|c|c|c|c|c|c|}
\hline \multirow[t]{2}{*}{ Compounds } & \multicolumn{2}{|l|}{ Regression equation } & \multicolumn{2}{|l|}{$R^{2}$} & \multicolumn{2}{|c|}{$\begin{array}{l}\text { LOD } \\
(\mu \mathrm{g} / \mathrm{mL})\end{array}$} & \multicolumn{2}{|c|}{$\begin{array}{l}\text { LOQ } \\
(\mu \mathrm{g} / \mathrm{mL})\end{array}$} & \multicolumn{2}{|c|}{$\begin{array}{l}\text { Precision, RSD (\%) } \\
\text { Intra-day }(n=3)\end{array}$} & \multicolumn{2}{|c|}{$\begin{array}{l}\text { Precision, RSD (\%) } \\
\text { Inter-day }(n=3)\end{array}$} \\
\hline & $\mathrm{TLC}^{*}$ & $\mathrm{UPLC}^{*}$ & TLC & UPLC & $\mathrm{TLC}$ & $\overline{U P L C}$ & $\overline{T L C}$ & $\overline{U P L C}$ & $\overline{T L C}$ & UPLC & $\overline{\mathrm{TLC}}$ & UPLC \\
\hline NR1 & $y=5752.1 x+796.27^{b}$ & $y=497892 x+1692^{a}$ & 0.993 & 0.994 & 16.60 & 3.77 & 49.80 & 11.30 & 3.17 & 1.95 & 1.04 & 4.59 \\
\hline $\mathrm{Rb} 1$ & $y=15159 x-498.43^{a}$ & $y=451285 x-8794.1^{a}$ & 0.991 & 0.997 & 12.60 & 4.26 & 37.90 & 41.70 & 7.06 & 1.67 & 0.92 & 0.55 \\
\hline $\mathrm{Rd}$ & $y=8877.8 x+1092.3^{b}$ & $y=502877 x+4048.3^{a}$ & 0.995 & 0.994 & 144.00 & 3.31 & 431.00 & 9.95 & 1.67 & 0.74 & 6.92 & 1.98 \\
\hline Rg1 & $y=8095.7 x+1360.3^{a}$ & $y=519908 x-3073.3^{a}$ & 0.992 & 0.993 & 126.00 & 16.20 & 378.00 & 48.80 & 2.23 & 3.58 & 4.68 & 2.82 \\
\hline Rg2 & $y=11839 x+958.33^{a}$ & $y=529865 x+1901.5^{a}$ & 0.991 & 0.998 & 33.700 & 5.97 & 101.00 & 17.90 & 0.77 & 2.18 & 2.80 & 0.26 \\
\hline
\end{tabular}

RSD $(\%)=100 \times$ S.D./mean; $y$, peak area; $x$, the concentration of each reference compound $(\mathrm{mg} / \mathrm{mL}) ; \mathrm{R}^{2}$, coefficient of determination; LOD, limit of detection $(3.33 \times(\mathrm{SD}$ of $\mathrm{Y}$-intercept/mean of slope) $)$; LOQ, limit of quantification $(10 \times(\mathrm{SD}$ of $\mathrm{Y}$-intercept/mean of slope $))$

${ }^{a} \mathrm{n}=3$

${ }^{\mathrm{b}} \mathrm{n}=4$

${ }^{*}$ All $P$ values for the regression equations are $=0.000$

Some of the granule products did not have the notoginseng content on the product label. One company explained that this information was not provided on the product label because it could vary from batch to batch. This variation could arise from collecting starting materials from different sources. Differences in the raw herbs would affect the amount of residue obtained after processing and extraction. The non-standardised manufacturing processes for granule products could affect the quality of the products and result in efficacy issues for practitioners and consumers.

\section{Validation of TLC and UPLC method Calibration, linearity, $L O Q$ and $L O D$}

The linear regression equations, coefficient of determination $\left(R^{2}\right)$, limit of quantification (LOQ), limit of detection (LOD), average intra- and inter-day repeatability (RSD) for each standard were shown in Table 2 . The $\mathrm{R}^{2}$ values were greater than 0.991 for all the analytes $(P$ values corresponding to a $R^{2}$ value of 0.000 ), showing good linearity for the experimental data for both analytical methods. The LODs for TLC and UPLC of the five marker compounds ranged from $12.60-144.00 \mu \mathrm{g} / \mathrm{mL}$ and $3.31-16.20 \mu \mathrm{g} / \mathrm{mL}$,

Table 3 Contents ( $\mathrm{mg} / \mathrm{g}$, mean $\pm \mathrm{SD}, \mathrm{n}=3$ ) of the five compounds in Sanqi samples analysed by TLC and UPLC-PDA

\begin{tabular}{|c|c|c|c|c|c|c|c|c|c|c|}
\hline \multirow[t]{2}{*}{ Sample } & \multicolumn{2}{|l|}{ NR1 } & \multicolumn{2}{|l|}{$\mathrm{Rb} 1$} & \multicolumn{2}{|l|}{$\mathrm{Rd}$} & \multicolumn{2}{|l|}{$\mathrm{Rg} 1$} & \multicolumn{2}{|l|}{$\mathrm{Rg} 2$} \\
\hline & TLC & UPLC & TLC & UPLC & TLC & UPLC & TLC & UPLC & TLC & UPLC \\
\hline R1 & $31.77 \pm 0.80$ & $6.06 \pm 0.97$ & $24.54 \pm 0.39$ & $17.82 \pm 1.38$ & $3.35 \pm 2.07$ & $4.02 \pm 0.23$ & $58.06 \pm 0.57$ & $38.70 \pm 1.51$ & $2.51 \pm 0.45$ & $1.83 \pm 0.12$ \\
\hline R2 & $41.64 \pm 1.47$ & $5.48 \pm 1.16$ & $27.38 \pm 0.36$ & $19.21 \pm 2.37$ & $8.36 \pm 0.32$ & $7.08 \pm 1.54$ & $41.78 \pm 0.81$ & $25.38 \pm 2.34$ & $4.59 \pm 0.24$ & $3.39 \pm 0.7$ \\
\hline R3 & $35.64 \pm 0.30$ & $6.05 \pm 0.92$ & $31.91 \pm 0.77$ & $17.78 \pm 0.78$ & $5.94 \pm 0.88$ & $6.82 \pm 0.12$ & $47.89 \pm 1.41$ & $33.87 \pm 0.93$ & $3.47 \pm 0.34$ & $2.56 \pm 0.0$ \\
\hline R4 & $27.15 \pm 1.09$ & $9.15 \pm 0.23$ & $18.23 \pm 0.99$ & $21.54 \pm 0.23$ & $11.84 \pm 0.65$ & $6.95 \pm 0.33$ & $53.80 \pm 2.65$ & $39.68 \pm 1.40$ & $7.57 \pm 0.10$ & $2.30 \pm 0.1$ \\
\hline R5 & $8.47 \pm 0.77$ & $6.20 \pm 0.55$ & $6.56 \pm 0.39$ & $12.79 \pm 0.38$ & ND & $6.00 \pm 0.41$ & $45.35 \pm 1.62$ & $35.71 \pm 0.73$ & ND & $2.38 \pm 0.2$ \\
\hline R6 & $3.03 \pm 0.04$ & $4.26 \pm 0.05$ & $3.73 \pm 0.10$ & $16.48 \pm 0.77$ & $2.77 \pm 0.06$ & $6.90 \pm 0.67$ & $10.55 \pm 0.19$ & $23.63 \pm 0.39$ & $1.87 \pm 0.07$ & $3.59 \pm 0.06$ \\
\hline G1 & $2.21 \pm 0.29$ & $0.84 \pm 0.16$ & $2.93 \pm 0.06$ & $3.03 \pm 0.23$ & $2.17 \pm 0.07$ & $0.80 \pm 0.03$ & $4.14 \pm 0.02$ & $4.00 \pm 0.34$ & $0.63 \pm 0.04$ & $0.32 \pm 0.0$ \\
\hline G2 & $3.77 \pm 0.06$ & $1.37 \pm 0.04$ & $4.49 \pm 0.32$ & $4.97 \pm 0.14$ & $1.20 \pm 0.26$ & $2.06 \pm 0.20$ & $8.83 \pm 0.40$ & $5.15 \pm 0.10$ & $2.68 \pm 0.16$ & $2.72 \pm 0.3$ \\
\hline G3 & $9.46 \pm 1.48$ & $2.46 \pm 0.03$ & $7.34 \pm 0.56$ & $9.74 \pm 0.09$ & $3.03 \pm 2.56$ & $2.50 \pm 0.06$ & $12.79 \pm 0.89$ & $12.94 \pm 0.14$ & $1.56 \pm 0.14$ & $0.57 \pm 0.0$ \\
\hline $\mathrm{G} 4$ & $0.86 \pm 0.13$ & $0.37 \pm 0.01$ & $1.24 \pm 0.14$ & $1.52 \pm 0.09$ & $1.28 \pm 0.33$ & $0.38 \pm 0.01$ & $4.13 \pm 0.48$ & $2.14 \pm 0.01$ & $0.58 \pm 0.03$ & $0.09 \pm 0.0$ \\
\hline G5 & $0.40 \pm 0.19$ & $0.52 \pm 0.01$ & $1.11 \pm 0.02$ & $2.22 \pm 0.01$ & $0.02 \pm 0.00$ & $0.57 \pm 0.01$ & $2.13 \pm 0.08$ & $2.91 \pm 0.04$ & $0.27 \pm 0.03$ & $0.09 \pm 0.0$ \\
\hline G6 & $2.51 \pm 0.05$ & $0.72 \pm 0.01$ & $1.40 \pm 0.15$ & $2.56 \pm 0.02$ & $0.64 \pm 0.04$ & $0.93 \pm 0.01$ & $5.14 \pm 0.02$ & $3.27 \pm 0.03$ & $0.26 \pm 0.05$ & $0.19 \pm 0.0$ \\
\hline G7 & $11.96 \pm 1.10$ & $7.86 \pm 0.41$ & $16.59 \pm 0.18$ & $32.93 \pm 2.01$ & $8.57 \pm 2.49$ & $9.52 \pm 1.53$ & $27.73 \pm 0.84$ & $42.95 \pm 2.73$ & $3.11 \pm 0.88$ & $1.63 \pm 0.2$ \\
\hline G8 & $0.02 \pm 0.01$ & $0.08 \pm 0.00$ & $0.36 \pm 0.01$ & $0.42 \pm 0.02$ & $0.35 \pm 0.01$ & $0.07 \pm 0.00$ & $0.58 \pm 0.01$ & $0.52 \pm 0.01$ & $0.08 \pm 0.01$ & $0.08 \pm 0.0$ \\
\hline G9 & $5.39 \pm 0.05$ & $2.83 \pm 0.07$ & $5.31 \pm 0.13$ & $10.60 \pm 0.43$ & $3.24 \pm 0.13$ & $2.47 \pm 0.40$ & $13.58 \pm 0.49$ & $15.14 \pm 0.59$ & $1.55 \pm 0.48$ & $0.57 \pm 0.0$ \\
\hline G10 & $5.81 \pm 0.25$ & $2.72 \pm 0.36$ & $5.59 \pm 0.14$ & $12.07 \pm 0.95$ & $1.48 \pm 0.41$ & $2.94 \pm 0.19$ & $13.50 \pm 0.08$ & $15.73 \pm 1.93$ & ND & $0.52 \pm 0.1$ \\
\hline G11 & $12.28 \pm 0.09$ & $1.16 \pm 0.19$ & $11.22 \pm 0.33$ & $4.20 \pm 0.62$ & $1.26 \pm 0.51$ & $1.71 \pm 0.57$ & $19.67 \pm 0.09$ & $4.65 \pm 0.90$ & ND & $1.98 \pm 0.03$ \\
\hline G12 & $11.72 \pm 0.71$ & $7.51 \pm 0.42$ & $12.67 \pm 0.03$ & $24.12 \pm 0.15$ & $7.58 \pm 0.54$ & $7.28 \pm 0.17$ & $30.26 \pm 0.95$ & $34.88 \pm 0.26$ & $2.56 \pm 0.08$ & $1.23 \pm 0.24$ \\
\hline
\end{tabular}


respectively. The LOQs for TLC and UPLC of the five marker compounds were 37.90-378.00 and 9.95$48.80 \mu \mathrm{g} / \mathrm{mL}$, respectively. The LODs and LOQs from UPLC were generally lower than those from TLC ( $P=0.016$ and $P=0.032$ for LOD and LOQ, respectively), indicating higher sensitivity was achieved with the UPLC method.

\section{Precision}

Good instrumental and method precision was obtained for both UPLC and TLC. As shown in Table 2, the RSD for the intra- and inter-day precision ranged from $0.768-7.059 \%$ and $0.916-6.916 \%$ for TLC. For UPLC, the corresponding ranges were $0.739-3.579 \%$ and $0.236-4.591 \%$, respectively.

\section{Quantification by TLC}

The resolution of TLC for the marker compound notoginseng NR1 and ginsenoside Re was not sufficient to quantify these compounds separately (Additional file 4). As the content of Re in notoginseng is reportedly approximately half that of NR1 [23], the total NR1 + Re peak was used for the quantification of NR1 in this study. The contents for the marker compounds detected in the samples were shown in Table 3. The following compounds were below the limit of quantification: Rg2 in samples R5, G10 and G11, and Rd in sample R5. Fluorescence (UV $366 \mathrm{~nm}$ ) was used for visualisation of the derivatised saponins, and this enhanced their detection compared with the lower absorbance measurement at $254 \mathrm{~nm}$. However, the noise level also increased [17]. Although the TLC profiles of the raw herbs and granule extracts were similar, the contents of the marker compounds were much higher in the raw herbs than in the granules, with the exception of G7 and G12.

\section{Quantification by UPLC-PDA}

Typical UPLC-PDA profiles of the raw notoginseng and granule extracts were shown in Fig. 1. The contents of the marker compounds in the samples were shown in Table 3. After many UPLC runs, ginsenosides Re and Rg1 could not be successfully separated. This was also a problem in previous chromatography studies [24-27]. According to Wan et al. [28], the Rg1/Re ratio was $6.19 \pm 0.82$ in 18 samples of notoginseng from different origins. As the content of Re is much smaller than that of Rg1 and
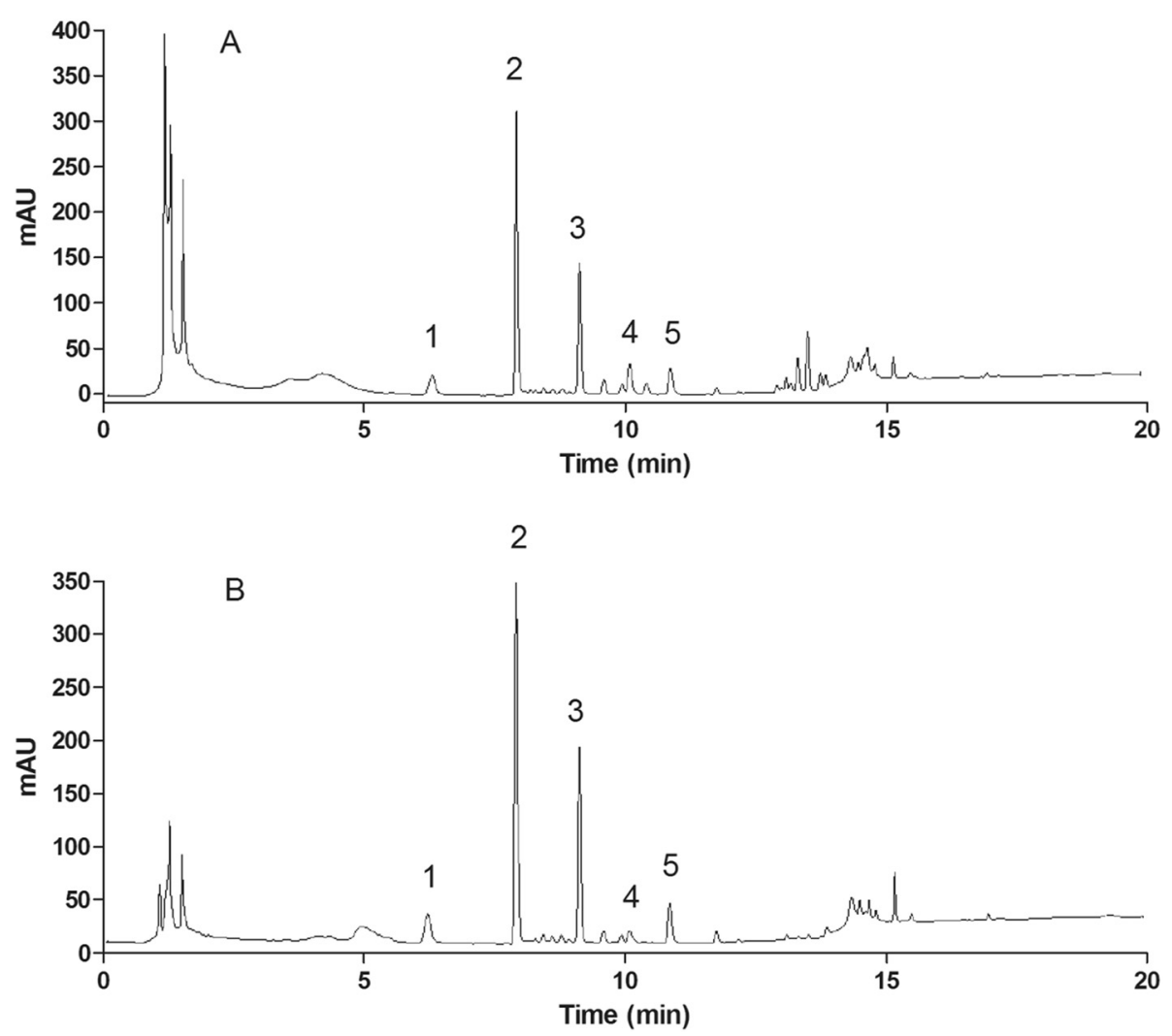

Fig. 1 Chromatograms of five ginsenosides analysed by UPLC-PDA. a Raw herb extract (R6), b Granule extract (G12). (1) NR1, (2) Rg1, (3) Rb1, (4) Rg2 and (5) Rd 
this is a comparative study, the total content of $\operatorname{Rg} 1$ and Re was used as the Rg1 content. The low standard deviations of the five standards in the raw herbs and granules showed good repeatability for the quantification (Table 3). The contents of the marker compounds in the granules were substantially lower than those in the raw herb samples, with the exception of G7 and G12. The contents of each of the five compounds were not significantly different within the raw herb samples and within the granule products $(P>0.05)$. However, these two groups were significantly different when the contents were compared $(P=0.000)$. Differences in the contents of the marker compounds in the granules might be related to the variable yields (Table 1) and the different notoginseng contents claimed by the manufacturers.

\section{Differentiations of raw notoginseng and granules}

The contents of the five marker compounds were used in HCA analysis for grouping of similar products (Fig. 2), and two groups were found. For TLC, the Cluster 1 included samples R1 to R4, and the Cluster 2 included R5 and $\mathrm{R} 6$ and the granule products. The clustering of R5 and R6 by HCA with the granule products could have arisen because the concentrations of some marker compounds in these samples were too low for TLC quantification.

For UPLC, HCA showed a similar pattern, and samples G7 and G12 were grouped with the raw herbs (Group 1) and the rest of the granules as Group 2.

PCA was also applied to differentiate samples by displaying them as coordinates in maps based on the contents of the five marker compounds. In the PCA biplot, each point represents an individual sample and the red line represents the contribution of each original variable to the score of two major PCs (Fig. 3). For TLC, PC1 represented up to $82.73 \%$ of the total variance and $\mathrm{PC} 2$ (10.64 \%) cumulatively explained up to $93.37 \%$ of total variance. The distribution of the samples based on PC1 was due to the variance from all the markers compounds, while the separation of samples by $\mathrm{PC} 2$ was based on the contents of $\mathrm{Rd}$ and $\mathrm{Rg} 2$. The TLC biplot differentiated the raw herbs and granules. Samples R1 to R4 (Cluster 1) showed higher contents of all the marker compounds than the other samples. In particular, R4 had the highest contents of Rd and Rg2. Samples R5 and R6 were plotted close to the granules (Cluster 2).

For UPLC, the biplot showed that PC1 (84.77 \%) and PC2 (11.46 \%) cumulatively explained up to $96.23 \%$ of

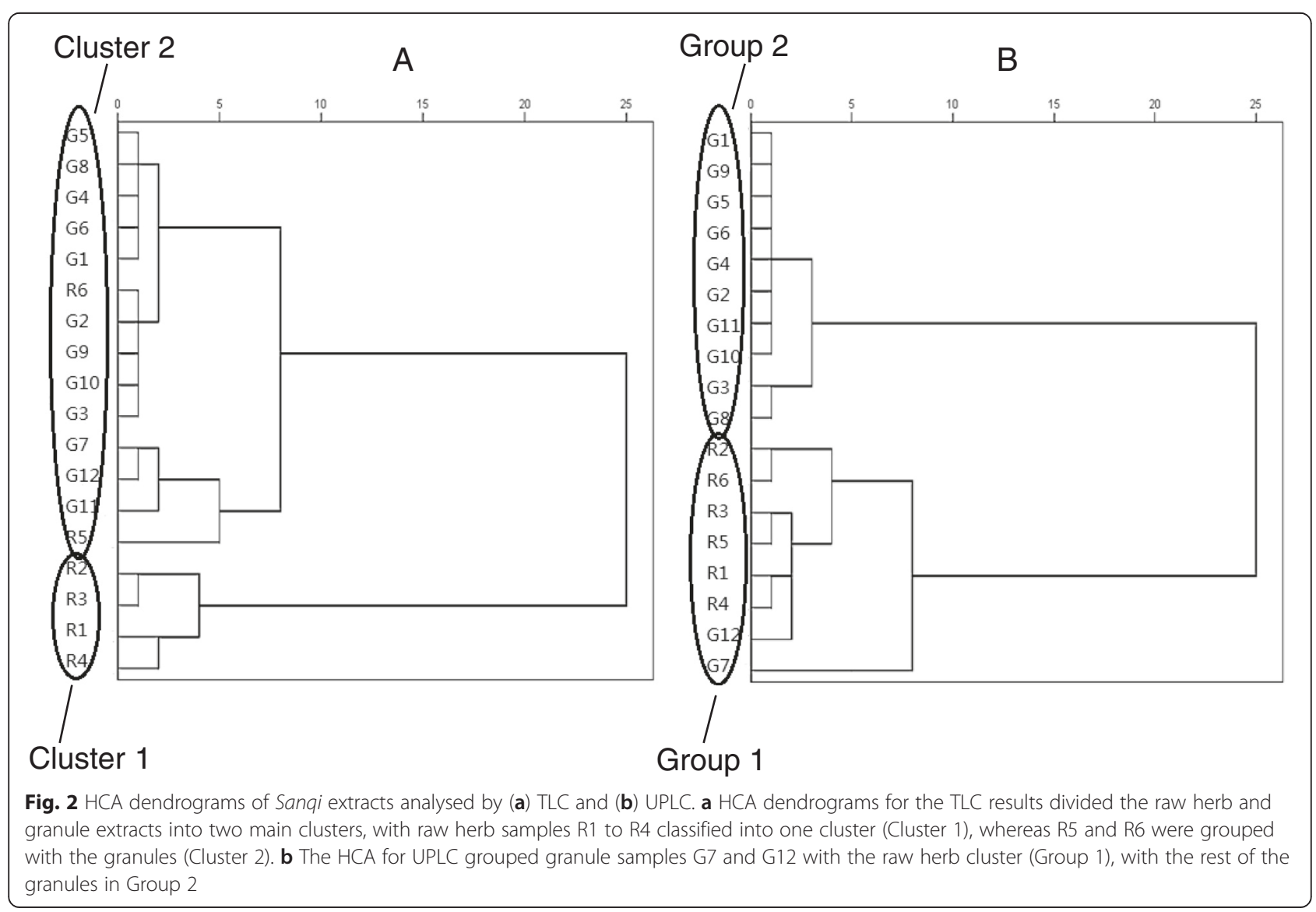




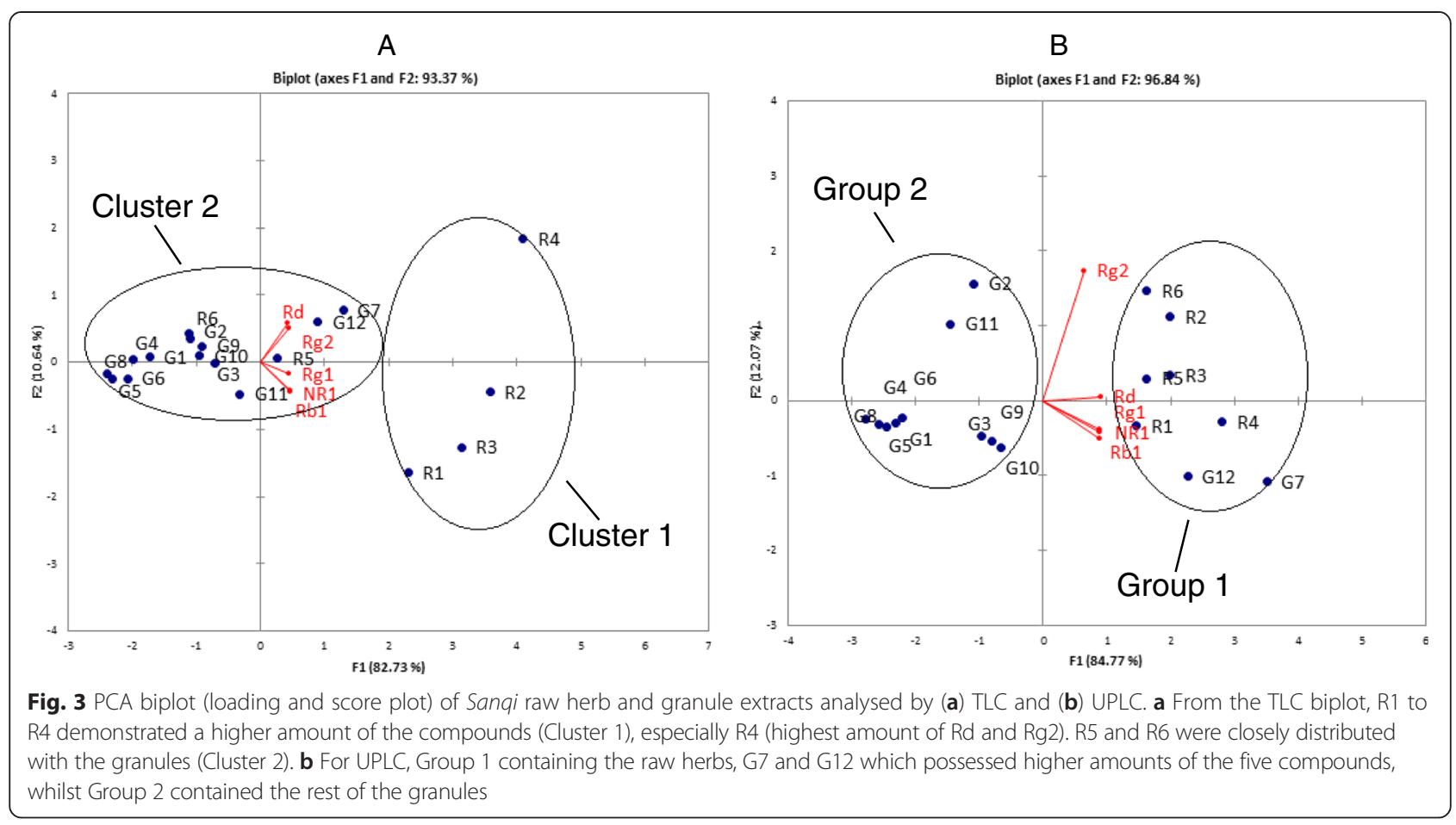

total variance. PC1 showed the variance from the original variables, and $\mathrm{PC} 2$ was mainly related to $\mathrm{Rg} 2$. Based on the two PCs, the PCA biplots for the UPLC results divided the samples into two groups: Group 1 contained the raw herbs and G7 and G12 of the granule products, whilst Group 2 contained the rest of the granule products. This agreed with the UPLC HCA results.

\section{Current pharmacopoeia standards for notoginseng}

In the PPRC, ginsenosides Rg1, Rb1 and notoginseng NR1 are recommended for the quality assessment of the raw herb [29]. The total ginsenosides content (i.e., NR1 + Rg1 + $\mathrm{Rb} 1$ ) in the root and rhizome of notoginseng (methanol extract) should not be less than $5.0 \%$. However, this guideline does not apply to granules [29]. From the TLC calculations (Table 4), all the raw herb samples (R1-R6) met this minimum requirement, with the total ginsenosides content ranging from $6.0 \%$ to $11.5 \%$. By contrast,

Table 4 Pearson correlation between the contents of the compounds analysed by UPLC and the anti-oxidant capacity of the extracts

\begin{tabular}{ll}
\hline Components & ABTS \\
\hline Rg2 & $0.740^{\mathrm{a}}$ \\
$\mathrm{Rd}$ & $0.836^{\mathrm{a}}$ \\
$\mathrm{Rb} 1$ & $0.772^{\mathrm{a}}$ \\
$\mathrm{NR} 1$ & $0.769^{\mathrm{a}}$ \\
$\mathrm{Rg} 1$ & $0.768^{\mathrm{a}}$ \\
\hline
\end{tabular}

${ }^{a}$ Correlation is significant at the 0.01 level (2-tailed) the total ginsenosides content in most of the granules was less than $5.0 \%$ (range $0.1 \%$ to $4.3 \%$ ). In the granules, the contents of G7 and G12 were high at $5.6 \%$ and $5.5 \%$, respectively.

From the UPLC data (Table 3), the raw herb samples (R1-R5) met the minimum requirement of PPRC, with total ginsenosides contents ranging from $5.0 \%$ to $8.2 \%$. Only sample R6 had a relatively low total ginsenosides content $(4.4 \%)$. As with the TLC results, most of the granules contained less than $5.0 \%$ of these three marker compounds, with total ginsenosides contents ranging from $0.1 \%$ to $3.1 \%$. Again, the contents of G7 and G12 were high at $8.4 \%$ and $6.7 \%$, respectively. Due to the content differences between raw notoginseng and granules, standards for the granules need to be established and included in future editions of the PPRC.

For comparative purposes, HCA was conducted using the contents of the three marker compounds (NR1, Rg1 and Rb1) specified by the PPRC [29]. From the TLC results, HCA produced clusters that were identical to those derived when using the contents of all five marker compounds studied. However, for the UPLC results, R2 and R6 were grouped with the granules, compared to Group 1 which included all the raw herbs plus G7 and G12 (Additional file 5). These results suggest that more marker compounds (i.e., five rather than three) are required to differentiate raw notoginseng from granule products [29]. A similar finding was reported for the roots of Pueraria lobata and P. thomsonii and their granule products [30]. 


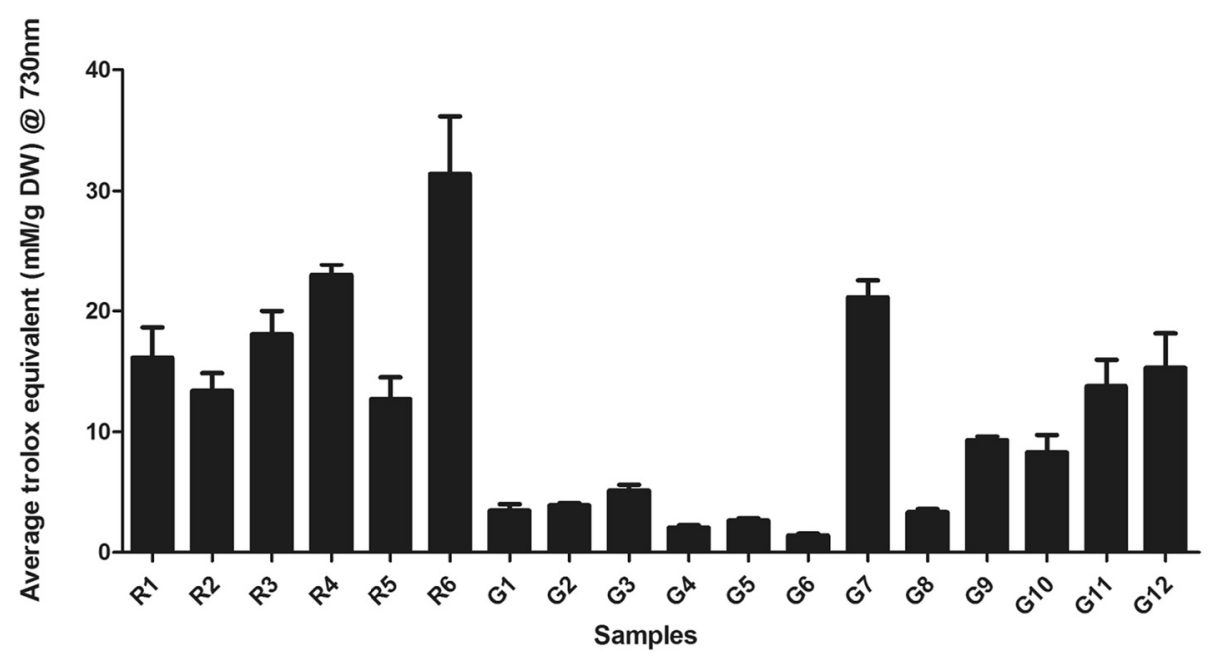

Fig. 4 ABTS activity of Sanqi raw herb and granule extracts $(n \geq 3)$. Generally, raw herb samples $(12.68-31.41 \mathrm{mM} / \mathrm{g}$ DW) showed a higher ABTS radical scavenging activity than granule samples $(1.41-21.13 \mathrm{mM} / \mathrm{g} D W)$. R6 exhibited the highest anti-oxidant activity among all the samples (31.41 mM/g DW). For the granule samples, G7 and G12 possessed strong radical scavenging activity (21.13 and 15.28 mM/g DW, respectively)

\section{ABTS assay for antioxidants}

The ABTS assay was conducted at both $730 \mathrm{~nm}$ and $410 \mathrm{~nm}$ for comparison with the literature, and both wavelengths gave similar results (Fig. 4). The results for the ABTS assay at $730 \mathrm{~nm}$ are reported here, as the values are reported as Trolox equivalents and this is measured at $730 \mathrm{~nm}$. R6 exhibited the highest antioxidant activity among all the samples $\left(31.41 \mathrm{mmol} \mathrm{L}^{-1} / \mathrm{g} \mathrm{DW}\right)$. For the granules, both G7 and G12 showed strong radical scavenging activity (21.13 and $15.28 \mathrm{mmol} \mathrm{L}^{-1} / \mathrm{g} \mathrm{DW}$, respectively). Non-parametric analysis showed that the antioxidant capacities of G7 and G12 were similar to R1-R6 (Group 1) $(P>0.05)$. The ABTS radical scavenging activity of Group 1 was significantly higher than that of Group $2(P=0.005)$. Pearson correlation was performed to examine the relationship between the quantitative data and radical scavenging capacities. There were significant correlations between the UPLC results for the marker contents and the corresponding antioxidant activities (Table 4).

\section{Conclusion}

UPLC was more efficient than TLC for the simultaneous determination of the five major compounds in Sanqi products in terms of linearity, higher sensitivity and repeatability. The statistical analysis of the samples by HCA and PCA revealed that contents of the marker compounds were significantly higher in the raw herb group than the granule group.

\section{Additional files}

Additional file 1: Chemical structures of the compounds assessed

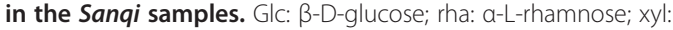
$\beta$-D-xylose [22].
Additional file 2: Information for Sanqi raw herbs and granule samples. Sanqi raw herbs were collected from six different sources in China and twelve herbal granule products were purchased from China, Taiwan and Australia.

Additional file 3: TLC of methanol extract and residue from the raw herb/granule. Lane 1: Methanol extract of granule sample 4 (G4); Lane 2: Residue of the methanol extract of G4; Lane 3: Water and methanol extract of the raw herb R1; Lane 4: Residue of water and methanol extract of raw herb. As shown in the figure, Lanes 1 and 3 of the methanol extract contain many bands which represented the marker compounds, whereas Lanes 2 and 4 were absent of the bands. This supported the assumption that methanol efficiently extracted the major compounds.

Additional file 4: TLC fluorescence image of Sanqi raw herb and granule samples with the standards under UV mode (366 nm). Key: Lane 1 - Notoginseng NR1; Lane 2 - Ginsenoside Re; Lane 3 - Ginsenoside Rg1; Lane 4 - R6 methanol extract; Lane 5 - G12 methanol extract; Lane 6 -Ginsenoside Rg2; Lane 7 - Ginsenoside Rd; Lane 8- Ginsenoside Rb1.

Additional file 5: HCA dendograms of Sanqi extracts analysed by (A) TLC and (B) UPLC results using three markers (NR1, Rg1 and $\mathbf{R b} 1)$. For TLC, HCA revealed that the clusters were identical to those derived from the content of the five marker compounds. However, for the HCA result analysed by UPLC, R2 and R6 were grouped with the granules.

\section{Abbreviations}

ABTS: 2,2'-azino-bis(3-ethylbenzothiazoline-6-sulphonic acid); HCA: Hierarchical component analysis; HPLC: High-performance liquid chromatography; LOD: Limitation of detection; LOQ: Limitation of quantification; NR1: Notoginsenoside R1; PC: Principal component; PCA: Principal component analysis; PPRC: Pharmacopoeia of the People's Republic of China; Rb1: Ginsenoside Rb 1; Rd: Ginsenoside Rd; Rg1: Ginsenoside Rg1; Rg2: Ginsenoside Rg2; RSD: Relative standard deviation; R² $^{2}$ Coefficient of determination; TLC: Thin layer chromatography; UPLC-PDA: Ultra-performance liquid chromatography with photo diode array.

\section{Competing interests}

The authors declare that they have no competing interests.

\section{Authors' contributions}

KC conceived the study. KC and VRN designed the study. XZ performed the experiments. XZ and VRN analysed the data. XZ drafted the manuscript. KC and VRN revised the manuscript. All authors read and approved the final manuscript. 


\section{Acknowledgements}

The project was supported by the Joint Chair in Traditional Chinese Medicine Program (New South Wales - Office of Science and Research, The University of Sydney and University of Western Sydney, Australia). Many thanks to Mr. James Hennell and Mr. Rohan Grace for technical training and assistance in TLC. We appreciate Mr. Jarryd Pearson for the technical support in UPLC instrumentation. We are grateful to Mr. John Truong and Mr. Antony Kam for their LC MS/MS and antioxidant technical assistance, respectively. Thank you to Mr. Paul Fahey for the ongoing statistical advice.

Received: 14 May 2014 Accepted: 7 May 2015

Published online: 06 June 2015

\section{References}

1. Brand E. A clinician's guide to using granule extracts. Boulder, Colo: Blue Poppy Press; 2010

2. Liang XM, Jin Y, Wang YP, Jin GW, Fu Q, Xiao YS. Qualitative and quantitative analysis in quality control of traditional Chinese medicines. J Chromatogr A. 2009;1216(11):2033-44.

3. Fan XH, Cheng YY, Ye ZL, Lin RC, Qian ZZ. Multiple chromatographic fingerprinting and its application to the quality control of herbal medicines. Anal Chim Acta. 2006;555(2):217-24.

4. Chen Y, Zhu SB, Xie MY, Nie SP, Liu W, Li C, et al. Quality control and original discrimination of Ganoderma lucidum based on high-performance liquid chromatographic fingerprints and combined chemometrics methods. Anal Chim Acta. 2008:623(2):146-56.

5. Liang YZ, Xie PS, Chan K. Quality control of herbal medicines. J Chromatogr B. 2004;812(1):53-70.

6. Z Zhang YY, Sun SJ, Dai JY, Wang WY, Cao HJ, Wu JB et al. Quality control method for herbal medicine-chemical fingerprint analysis. In: Shoyama $Y$, editor. Quality control of herbal medicines and related areas. Croatia: In Tech; 2011. p. 171-94.

7. Kong WJ, Zhao YL, Xiao XH, Jin C, Li ZL. Quantitative and chemical fingerprint analysis for quality control of Rhizoma Coptidischinensis based on UPLC-PAD combined with chemometrics methods. Phytomedicine. 2009;16(10):950-9.

8. Wang XZ, Yang Y, Li R, McGuinnes C, Adamson J, Megson IL, et al. Principal component and causal analysis of structural and acute in vitro toxicity data for nanoparticles. Nanotoxicology. 2014;8(5):465-76.

9. China Food and Drug Administration database. http://app1.sfda.gov.cn/ datasearch/face3/base.jsp?tableld=25\&tableName=TABLE25\&title=\%B9\% FA\%B2\%FA\%D2\%A9\%C6\%B7\&bcld=1243565603038869090157374477882. Accessed 18 June 2014.

10. Ng T. Pharmacological activity of sanchi ginseng (Panax notoginseng). J Pharm Pharmacol. 2006;58(8):1007-19.

11. Wang J, Huang Z, Cao H, Wang Y, Hui P, Hoo C, et al. Screening of anti-platelet aggregation agents from Panax notoginseng using human platelet extraction and HPLC-DAD-ESI-MS/MS. J Sep Sci. 2008;31(6-7):1173-80.

12. White CM, Fan C, Song J, Tsikouris JP, Chow M. An evaluation of the hemostatic effects of hydrophilic, alcohol, and lipophilic extracts of notoginseng. Pharmacotherapy. 2001;21(7):773-7.

13. Zhang W, Wojta J, Binder BR. Effect of notoginsenoside R1 on the synthesis of tissue-type plasminogen activator and plasminogen activator inhibitor-1 in cultured human umbilical vein endothelial cells. Arterioscl Throm Vas. 1994;14(7):1040-6.

14. Tipoe GL, Leung T-M, Hung M-W, Fung M-L. Green tea polyphenols as an anti-oxidant and anti-inflammatory agent for cardiovascular protection. Cardiovasc Hematol Dis Drug Targets. 2007;7(2):135-44.

15. Russo C, Olivieri O, Girelli D, Faccini G, Zenari ML, Lombardi S, et al. Anti-oxidant status and lipid peroxidation in patients with essential hypertension. J Hypertens. 1998;16(9):1267-71.

16. CAMAG. APPLICATION NOTES In: HPTLC Identification of Notoginseng (Panax notoginseng or Panax pseudoginseng) CAMAG TLC/HPTLC METHODS. 2008. http://www.camag.com/en/tlc_hptlc/camag_laboratory/ methods.cfm. Accessed 05 September 2013.

17. Fuzzati N. Analysis methods of ginsenosides. J Chromatography B. 2004:812(1):119-33.

18. Guan J, Lai CM, Li SP. A rapid method for the simultaneous determination of 11 saponins in Panax notoginseng using ultra performance liquid chromatography. J Pharm Biomed Anal. 2007;44(4):996-1000.
19. Chan C, Lee $Y$, Lam H, Zhang $X$. Analytical method validation and instrument performance verification. New York: Wiley; 2004.

20. Arnao MB, Cano A, Acosta M. The hydrophilic and lipophilic contribution to total antioxidant activity. Food Chem. 2001;73(2):239-44.

21. Ozgen M, Reese RN, Tulio AZ, Scheerens JC, Miller AR. Modified 2, 2-azino-bis-3-ethylbenzothiazoline-6-sulfonic acid (ABTS) method to measure antioxidant capacity of selected small fruits and comparison to ferric reducing antioxidant power (FRAP) and 2, 2'-diphenyl-1-picrylhydrazyl (DPPH) methods. J Agric Food Chem. 2006;54(4):1151-7.

22. Dan M, Su M, Gao X, Zhao T, Zhao A, Xie G, et al. Metabolite profiling of Panax notoginseng using UPLC-ESI-MS. Phytochemistry. 2008;69:2237-44.

23. Lau AJ, Woo SO, Koh HL. Analysis of saponins in raw and steamed Panax notoginseng using high-performance liquid chromatography with diode array detection. J Chromatogr A. 2003;1011(1):77-87.

24. Vanhaelen Fastre RJ, Faes ML, Vanhaelen MH. High-performance thin-layer chromatographic determination of six major ginsenosides in Panax ginseng. J Chromatogr A. 2000;868(2):269-76.

25. Chan EC, Yap SL, Lau AJ, Leow PC, Toh DF, Koh HL. Ultra-performance liquid chromatography/time-of-flight mass spectrometry based metabolomics of raw and steamed Panax notoginseng. Rapid Commun Mass Spectrom. 2007;21(4):519-28

26. Toh DF, New LS, Koh HL, Chan ECY. Ultra-high performance liquid chromatography/time-of-flight mass spectrometry (UHPLC/TOFMS) for time-dependent profiling of raw and steamed Panax notoginseng. J Pharm Biomed Anal. 2010;52(1):43-50.

27. Wan JB, Li P, Li S, Wang Y, Dong TTX, Tsim KWK. Simultaneous determination of 11 saponins in Panax notoginseng using HPLC-ELSD and pressurized liquid extraction. J Sep Sci. 2006;29(14):2190-6.

28. Wan JB, Sp L, Chen JM, Wang YT. Chemical characteristics of three medicinal plants of the Panax genus determined by HPLC-ELSD. J Sep Sci. 2007;30(6):825-32.

29. National Pharmacopoeia Committee. Chinese pharmacopoeia. Beijing: China Medical Science Press; 2010.

30. Wong KH, Razmovski-Naumovski V, Li KM, Li GQ, Chan K. Differentiation of Pueraria lobata and Pueraria thomsonii using partial least square discriminant analysis (PLS-DA). J Pharm Biomed Anal. 2013;84:5-13.

\section{Submit your next manuscript to BioMed Central and take full advantage of:}

- Convenient online submission

- Thorough peer review

- No space constraints or color figure charges

- Immediate publication on acceptance

- Inclusion in PubMed, CAS, Scopus and Google Scholar

- Research which is freely available for redistribution

Submit your manuscript at www.biomedcentral.com/submit 